\title{
L'avenir et la place de la construction de petites centrales hydrauliques en France
}

\author{
Small hydropower stations building future and situation in France
}

\author{
par J. - P. Corbet
}

Syndicat des constructeurs de petites turbines hydrauliques

Nowadays, few new small hydropower stations are built in France. The french turbine manufacturers export the major part of their products and lack recent references in their country.

\section{I ם UN MARCHÉ ACTUELLEMENT ORIENTÉ À L'EXPORTATION}

La profession de la petite hydraulique en France existe ; elle existe depuis 100 ans, date des premières créations, elle existe malgré les réglementations de toute nature, elle contribue au solde positif de la balance commmerciale de la France.

Avec plus de $85 \%$ de ventes de turbines à l'exportation, la profession progresse sur tous les marchés avec une adaptation rare aux ouvertures et aux contraintes réglementaires des pays dans lesquels l'hydroélectricité est une énergie potentielle.

Certains pays, en effet, à la faveur d'un prix de rachat de l'électricité plus intéressant, ou d'une politique énergétique décidée par un gouvernement, vont faire appel aux compétences techniques des fabricants de turbines qui souvent se chargent du montage de l'ensemble mécanique.

Il faut alors que les turbiniers puissent proposer leurs solutions rapidement car les politiques axées sur la diversité des sources d'énergie sont généralement suivies, une dizaine d'années après, par une réglementation environnementale plus tâtillone, une politique contraire favorisant d'autres types d'énergie... et le marché se referme.

Donc, une grande " écoute du marché " mondial, une flexibilité commerciale à l'affût des ouvertures de marchés sont généralement les paramètres des fabricants de turbines.

On comprendra, dans ce contexte, que les industriels français qui basent leur savoir-faire sur un accroissement des performances acquises depuis plusieurs décennies représentent entre 6 et $8 \%$ du marché mondial parmi les $35 / 40$ concurrents internationaux ou locaux à travers le monde.
Chaque constructeur dispose de listes de références nombreuses totalisant 2000 installations dans le monde depuis 25 ans, induisant un chiffre d'affaires annuel de toute la filière (incluant les fabricants, bureaux d'études spécialisés, soustraitants, experts hydrauliciens) de 1 milliard de francs et plus de 1000 emplois directs.

Voici pour le côté " face " de notre industrie. Mais il faut regarder le côté " pile".

La raison pour laquelle le chiffre " exportation " est important tient au manque de marché français, perturbé, voire anéanti par des réglementations élevées par les autorités françaises.

Rares sont les cours d'eau français qui ne soient pas “ classés ", rares sont les autorisations données pour de nouvelles installations.

La danger, à terme, viendra du fait qu'aucun turbinier français ne pourra montrer à des étrangers intéressés, des installations neuves. Nous allons perdre notre marché national, notre vitrine.

Pour prendre une image un peu simpliste, j'en conviens, mais très parlante, c'est comme si PSA et Renault ne pouvaient plus vendre de voitures en France et devaient de ce fait, se maintenir dans le peloton de tête des constructeurs mondiaux en exportant totalement l'ensemble de leur production. Impensable, irréaliste, car à terme, les marchés se fermeraient sur eux, le marché national ne serait plus porteur et le choix finalement pour eux consisterait à délocaliser complètement leurs services ou à mourir.

Tout le dialogue entamé conjointement avec les syndicats de producteurs (EAF, GPAE, Ecowatt par exemple) vise à convaincre les autorités de l'existence de cette industrie. Mais il y a encore beaucoup d'eau à turbiner avant de convaincre !!! 


\section{LA GRANDE TECHNICITÉ DE CETTE PROFESSION}

Quoi de plus simple pour un " mécanicien " ou un " chaudronnier " que de faire un moulin ou une roue à aubes, voire de reproduire ce qui existe, une Pelton, une Kaplan, une Francis.

Pour maintenir une avance technologique, les fabricants se sont lancés, ensemble, dans des programmes de recherche et développement extrêmement importants dont voici quelques exemples :

- études sur les huiles biodégradables (vers des turbines propres),

- possibilité d'utiliser à terme des matériaux composites,

- éviter la cavitation des éléments pompes et turbines,

- maîtrise de la technique hydraulique par un accroissement des performances, et donc du rendement des installations,

- diminution du bruit des installations par l'étude d'impact de chaque élément de l'installation,

- enfin, une étude sur l'amélioration de la maintenance prédictive intéressant tous les producteurs de petites centrales hydroélectriques.

\section{III $\square$ L'AVENIR DES PETITES CENTRALES HYDROÉLECTRIQUES}

L'avenir de l'énergie issue des $\mathrm{PCH}$ est prometteur dans le monde. Les besoins en énergie seront considérables à long terme, compte tenu de l'augmentation de la population mondiale. Nul doute que la place de la petite hydroélectricité, toutes les études le montrent, sera importante par rapport aux autres énergies. Pour l'éolien, le Danemark est une exception puisque les côtes s'y prêtent ( $8 \%$ de l'énergie du pays) mais les autres pays, la France en particulier, n'assurent pas un développement durable et important de cette énergie.

L'énergie solaire en est encore à l'état expérimental et n'est pas rentable sur une grande partie du globe. L'Avenir est donc prometteur à l'exportation.

\section{LISTE DES TURBINIERS FRANÇAIS ADHÉRANT AU SCPTH}

- Alstom Minihydro (Grenoble, 38) : turbines hydrauliques de 1 à $15 \mathrm{MW}$, vannes et équipements connexes, installations complètes.

- Bouvier Hydro (Fontaine, 38) : Turbines hydrauliques de 1000 à $15000 \mathrm{~kW}$, vannes et équipements connexes, installations complètes.

- ESAC Energie (Saint Loup sur Semouse, 70) : Turbines hydrauliques de moins de $1000 \mathrm{~kW}$, turbines hydrauliques de 1000 à $15000 \mathrm{~kW}$, vannes et équipements connexes, installations complètes.

- Mecamidi (Toulouse, 31) : Turbines hydrauliques de moins de $1000 \mathrm{~kW}$, turbines hydrauliques de 1000 à $15000 \mathrm{~kW}$, installations complètes.

- THEE (Toul, 54) : Turbines hydrauliques de moins de $1000 \mathrm{~kW}$, installations complètes.

Pour la situation française, les " complicateurs " et les " réglementeurs" se sont associés pour que l'avenir de cette industrie soit compromis.

Reste que les techniques de plus en plus pointues dans le domaine de l'hydraulique permettent d'espérer des accroissements de rendement des installations existantes. Reste également que des exemples donnés par des adhérents montrent que des installations ont gagné environ $20 \%$ à la suite d'un changement des équipements d'une installation ancienne.

En effet, à défaut de l'installation de sites nouveaux, les techniques actuelles permettent donc d'améliorer la rentabilité de la petite hydroélectricité en changeant les éléments mécaniques. C'est un point positif.

Autre point de comparaison positif, des études récentes font apparaître qu'une $\mathrm{PCH}$ de $1 \mathrm{MW}$, par rapport à un groupe électrogène de même puissance électrique développée par ailleurs éminemment polluant, a, sur une durée de 10 ans, un coût total trois fois moindre.

De quoi faire réfléchir les investisseurs... 